\title{
REFLEXIÓN
}

\section{EJERCICIOS QUE APOYAN EL FUNCIONAMIENTO FISICO EN ADULTOS MAYORES CON SARCOPENIA}

\author{
MEDINA-FERNÁNDEZ ISAÍ ARTURO, ${ }^{1}$ TORRES-OBREGÓN REYNA ${ }^{2 *}$, ESPARZA-GONZÁLEZ \\ SANDRA CECILIA ${ }^{3}$, DELABRA-SALINAS MARÍA MAGDALENA ${ }^{4}$
}

\section{RESUMEN:}

ntroducción: La Sarcopenia es un problema de salud asociado al aumento de adultos mayores con poca o nula actividad física y cuyos efectos están relacionados con la funcionalidad, esta enfermedad es diagnosticada cuando existe una disminución de la masa, fuerza y menor desempeño físico. Actualmente, el ejercicio físico es una de las mejores opciones terapéuticas contra la sarcopenia. Desarrollo: Se describen dos puntos, el primero es el rol de ejercicio en la sarcopenia, el cual describe la importancia y beneficios del ejercicio en pacientes con esta enfermedad; el segundo punto son los tipos de ejercicio para la prevención de sarcopenia en este apartado se describen los ejercicios identificados como el entrenamiento progresivo de fuerza, ejercicios vibratorios, aeróbicos, de flexibilidad y equilibrio. Conclusión: Las intervenciones de ejercicios en adultos mayores con sarcopenia causa cambios significativos en la fuerza muscular, mejoramiento de la función física, masa muscular, extensión de la rodilla, marcha, equilibrio, así como en la velocidad de marcha.

Palabras clave: Sarcopenia, Anciano, Terapia por ejercicio, Atención de enfermería (DeCS, BIREME).

\section{ABSTRACT}

Introduction: Sarcopenia is a health problem associated with the increase of elderly adults with little or no physical activity and whose effects are related to functionality, this disease is diagnosed when there is a decrease in mass, strength and less physical performance. Currently, physical exercise is one of the best therapeutic options against sarcopenia. Development: Two points are described, the first one is the role of exercise in sarcopenia, which describes the importance and benefits of exercise in patients with this disease; the second point is the type of exercises for sarcopenia prevention, in this section the exercises identified such as progressive strength training, vibratory, aerobic, flexibility and balance exercises are described. Conclusion: Exercise interventions on elderly patients with sarcopenia cause significant changes in muscle strength, improvement of physical function, muscle mass, knee extension, march, balance, as well as march speed.

Key words: Sarcopenia, Elderly, Exercise Therapy, Nursing care (DeCS, BIREME).

'Licenciado en Enfermería, Facultad de Enfermería "Dr Santiago Valdés Galindo" Universidad Autónoma de Coahuila.

2Doctora en Ciencias de Enfermería, Facultad de Enfermería "Dr Santiago Valdés Galindo" Universidad Autónoma de Coahuila. E-mail: reyna_89_12@hotmail.com

${ }^{3}$ Doctora en Ciencias, Facultad de Medicina Universidad Autónoma de Coahuila

${ }^{4}$ Maestra en Ciencias de Enfermería, Facultad de Enfermería "Dr Santiago Valdés Galindo" Universidad Autónoma de Coahuila 


\section{INTRODUCCIÓN}

El envejecimiento es el proceso fisiológico que causa cambios en las especies durante el ciclo de vida, donde una de las modificaciones son la composición corporal, como en la masa muscular, masa grasa y la grasa visceral (1). Al disminuir la masa muscular se producen cambios en la fuerza y la funcionalidad, lo anterior se encuentra relacionado con la sarcopenia, misma que es diagnosticada a partir de la disminución de la masa muscular baja, fuerza muscular y menor rendimiento físico (2).

La sarcopenia es la pérdida de músculo, que de igual manera afecta a la fuerza y funcionalidad, influyendo en la calidad de vida del Adulto Mayor (AM), lo que ocasiona un impacto biológico, psicológico y social ${ }^{(1,3)}$. Al mismo tiempo, con el aumento de la población AM, será considerado un problema de salud pública, debido que en el mundo la prevalencia es del 10\%; afectando en promedio del 5 al $13 \%$ de los AM cuando su edad es mayor de 60 años y llega hasta en un $50 \%$ cuando la edad es mayor a 80 años $^{(4-6)}$.

El aumento de la población del AM y con base a la prevalencia de la sarcopenia, diversos países en su sistema de salud consideran que el enfoque en las patologías debe ser preventivo, efectivo, seguro y eficiente ${ }^{(7)}$. Acorde con esta ideología, surge la importancia de la enfermería en el cuidado del paciente con sarcopenia mediante la preservación de la funcionalidad del adulto mayor, con base en la actividad física, dieta y los suplementos nutricionales, con el fin de disminuir los problemas de salud ${ }^{(8)}$.

No obstante, es necesario la formulación de los cuidados antes mencionados para que sea aplicativos y adecuados en la práctica diaria, dichos cuidados pueden asegurarles una vida digna. Sin embargo, los hábitos poco saludables y la falta de ejercicio siguen siendo frecuentes entre los $\mathrm{AM}$, lo que ocasiona grandes problemas de salud en esta población ${ }^{(9,10)}$.

Actualmente, una de las opciones terapéuticas que ha sido más efectiva para la sarcopenia es el ejercicio físico, debido a que favorece el aumento de la masa y función muscular, a su vez es importante destacar que la edad no es una barrera para su realización y por ende puede llevarse a cabo la recuperación con éxito ${ }^{(11)}$. Tal efecto, el ejercicio es un método probado y seguro para mantener la condición física, además revierte los cambios en la composición corporal causados por el envejecimiento, como por ejemplo la pérdida del sistema músculo esquelético ${ }^{(12)}$.

De igual manera, el ejercicio es efectivo para prevenir la discapacidad y riesgo de caídas en el AM, siendo los resultados favorables para este grupo poblacional ${ }^{(13,14)}$. En relación con lo anterior, es necesario conocer los diversos tipos de ejercicio que puede ser realizado por la profe- sional enfermería, cuya intervención influirá en la prevención de la sarcopenia, así como mejorar la capacidad funcional, riesgo de caídas entre otros efectos positivos. Por ello se planteó el objetivo de describir los ejercicios que apoyan en el funcionamiento físico en adultos mayores con sarcopenia en el primer nivel de atención.

\section{DESARROLLO}

\section{El rol del ejercicio en la sarcopenia desarrollado me- diante intervenciones de enfermería}

Diversos estudios condicionan la falta de actividad física con diversos tipos de comorbilidad, el nivel de actividad física juega un papel importante para el desarrollo como en la prevención de la sarcopenia; actualmente varios programas de ejercicio físico aseguran una ganancia muscular progresivamente ${ }^{(15,16)}$. Por ello, es importante considerar siempre en la evaluación geriátrica lo relacionado con la funcionalidad, para la incorporación de conductas que contribuya en mantener y/o recuperar la autonomía ${ }^{(17)}$.

Así mismo, el ejercicio aumenta el tamaño del músculo, de esta manera la realización de la misma es una forma de prevención de la sarcopenia para evitar la limitación de las actividades de la vida diaria de los $\mathrm{AM}^{(18)}$. Así mismo, ocasiona una estimulación muscular, es decir tiene efectos protectores a nivel muscular ${ }^{(19)}$.

Por otra parte, el personal de enfermería debido a su contacto directo y frecuente con los pacientes y su capacidad para desarrollar relaciones terapéuticas de confianza, puede ayudar a motivar a los pacientes a realizar cambios en el comportamiento de salud, como el aumento de la actividad física ${ }^{(20)}$, estas intervenciones son con base a las clasificaciones de intervenciones en enfermería (NIC) ${ }^{(25)}$, mismas que incluyen en la tabla 1.

\section{Tipo de ejercicio para la prevención de sarcopenia}

Existen diversos tipos de ejercicio que permiten mejorar la función física de los AM y con respecto a los artículos analizados son el entrenamiento progresivo de fuerza, equilibrio, flexibilidad, aeróbicos y vibratorios. Para ello en los siguientes subtítulos se describen cada una de las modalidades, así como los estudios que han utilizado el ejercicio como intervención en la sarcopenia.

\section{Entrenamiento progresivo de fuerza}

Dentro de los tratamientos para prevenir la sarcopenia se encuentra el entrenamiento de la fuerza ${ }^{(13)}$, mismos que se puede contrarrestar deficiencias funcionales asociadas con la edad, y producir un incremento de la misma, mostrando que la capacidad músculo esquelética no se ve mermada por los años, ya que las tensiones musculares producen limitación en la movilidad. Por otro lado, este tipo de ejercicio permite trabajar ante resistencias 
Tabla 1. Cuidados que apoyan al control de la actividad física y ejercicio en la sarcopenia

\begin{tabular}{|l|l|}
\hline \multicolumn{2}{|c|}{ Intervención de enfermería } \\
\hline Enseñanza ejercicio prescrito 5612 & $\begin{array}{l}\text { La enseñanza del ejercicio produce cambios en el comportamiento del } \\
\text { adulto mayores que no ejercicio, obteniendo los conocimientos que in- } \\
\text { duzcan a adquirir nuevas aptitudes ante la vida, mejorar estilo de vida e } \\
\text { identificar los beneficios (21). }\end{array}$ \\
\hline Fomento del ejercicio 0200 & $\begin{array}{l}\text { El ejercicio permite prevenir la pérdida del sistema músculo esquelético } \\
\text { (12), siendo esta intervención la base del profesional de enfermería y del } \\
\text { paciente para observar los beneficios en su realización. }\end{array}$ \\
\hline $\begin{array}{l}\text { Fomento del ejercicio: entrenamiento } \\
\text { de fuerza 0201 }\end{array}$ & $\begin{array}{l}\text { El entrenamiento de fuerza es una estrategia para la prevención y trata- } \\
\text { miento de la sarcopenia, cuya realización mejora la capacidad funcional } \\
\text { del AM (22). }\end{array}$ \\
\hline $\begin{array}{l}\text { Fomento del ejercicio: estiramiento } \\
0202\end{array}$ & $\begin{array}{l}\text { Los ejercicios de estiramiento influyen en un cambio positivo de ejercicio } \\
\text { de las extremidades inferiores en los adultos mayores (23). }\end{array}$ \\
\hline Terapia de ejercicio: equilibrio & $\begin{array}{l}\text { El ejercicio de equilibrio ofrece un efecto beneficioso en la función física } \\
\text { como la mejora del desempeño físico y disminución del riesgo de caídas } \\
(24) .\end{array}$ \\
\hline
\end{tabular}

Fuente: Clasificaciones de intervenciones en enfermería (NIC), 2013.

con menores porcentajes de riesgo de aumentar la frecuencia cardiaca y cifras de la tensión arterial|(11).

Así mismo, está demostrado que produce un incremento de la masa muscular y potencia muscular, esto es debido a que induce la secreción de hormonas anabólicas ${ }^{(18)}$. De igual manera, causa un aumento en la fuerza del 107$227 \%$, aumento en el área muscular en un $11 \%$ y aumento de las fibras tipo I y II (34 y $28 \%$, respectivamente). También, se ha observado reducción de la grasa corporal, aumento de la densidad ósea, ${ }^{(26)}$. En relación con la intensidad y frecuencia del ejercicio, no se sabe cuál es la intensidad ideal para causar hipertrofia muscular; sin embargo, la evidencia refiere que este tipo de ejercicio en intensidades elevadas permite obtener dichos cam$\operatorname{bios}^{(27)}$.

Diversos estudios refieren la efectividad de este tipo de ejercicio, uno de ellos es el realizado en Brasil a mujeres mayores de 65 años con riesgo de sarcopenia, cuyo entrenamiento de fortalecimiento muscular permitió mejorar la capacidad, función y fuerza muscular ${ }^{(28)}$. Así mismo en Italia, se realizó un ensayo clínico controlado en hombres con diagnóstico de sarcopenia, siendo parte del tratamiento, el entrenamiento de resistencia en 12 semanas, donde se obtuvo una diferencia significativa de la fuerza muscular ${ }^{(29)}$. Sin embargo, un estudio realizado en Japón a mujeres de 70 años con obesidad sarcopénica, refirieron que los ejercicios de fuerza al combinarse con una buena alimentación tienen efectos beneficiosos sobre la composición corporal, componentes de la sangre y función física, así como mejora la masa muscular ${ }^{(30)}$.

\section{Ejercicios vibratorios}

Las evidencias demuestran que el entrenamiento de vibraciones incrementa la fuerza muscular, especialmente en los adultos mayores ${ }^{(27)}$. Además, este tipo de ejercicio es seguro, adecuado y eficaz para esta población, pero con resultados similares al ejercicio de resistencia convencional( ${ }^{(31)}$. Por otra parte, los estudios centrados en la efectividad recomiendan este tipo de ejercicio como modo terapéutico para la prevención y/o mejora de la sarcopenia, osteoporosis y otros problemas de salud, esto es porque mejora principalmente la coordinación inter e intramuscular ${ }^{(32)}$.

Este tipo de entrenamiento estimulan al cuerpo, mediante el aumento la carga gravitatoria, carga capaz de producir una hipertrofia muscular en personas de edad avanzada, lográndose mostrar algunos beneficios adicionales como la mejora del equilibrio ${ }^{(11)}$. Por otra parte, la aplicación de diferentes estudios refiere que este tipo de ejercicio deberían prescribirse de forma personalizada ${ }^{(31)}$.

En relación con lo anterior, se puede observar en el estudio realizado en España en adultos mayores de 65 años, cuya intervención fue de 11 semanas, no tuvo efectos 
hipertróficos en la masa muscular. Sin embargo, señalan que puede ser una buena terapia no farmacológica para la sarcopenia debido a que mejora la fuerza muscular. Otra investigación realizada en Bélgica realizado en mujeres mayores de 70 años confirma lo anteriormente referido ${ }^{(33,34)}$. No obstante, en Hong Kong, aplicaron una intervención de entrenamiento de vibración de cuerpo entero durante 11 semanas a adultos mayores con perdida muscular, indicaron que fue efectivo en la extensión de la rodilla a $180^{\circ}$ y $60^{\circ}$, así como para preservar el rendimiento muscular en la población ${ }^{(35)}$.

\section{Ejercicio de flexibilidad y equilibrio}

Estos dos tipos de ejercicios permiten que los músculos y los tejidos mantengan su estructura en el cuerpo ${ }^{(36)}$. En este sentido, los ejercicios de flexibilidad son los que permiten moverse través de un rango completo de movimiento, con el objetivo de disminuir riesgo de caídas y contribuir a los cambios del envejecimiento, en dicho caso puede ser estiramiento estático, dinámico, activo o combinado ${ }^{(37)}$.

Uno de los ejemplos de entrenamiento de equilibrio incluye caminar tándem, es decir juntar los pies y talones, así como caminar sobre una superficie dócil como colchones de espuma, otro ejemplo es mantener el equilibrio en equipos en movimiento ${ }^{(37)}$. Por ende, no hay recomendaciones específicas sobre frecuencia específica, intensidad o tipo de ejercicios de equilibrio para adultos mayores. Preferiblemente, los adultos mayores con riesgo de caídas deben equilibrar el entrenamiento tres o más días a la semana, sin embargo, los ejercicios pueden aumentar en dificultad al pasar de sostenerse en un soporte estable mientras se realizan los ejercicios hasta hacerlos sin apoyo ${ }^{(38)}$.

Por otra parte, la flexibilidad se va permeando al paso de los años y se acentúa por las modificaciones en el sistema óseo, así como en el acortamiento de los tendones y debilidad muscular ${ }^{(39)}$. En este sentido los ejercicios flexibilidad, no han demostrado tener un impacto sobre el volumen de la masa muscular, así como en la fuerza, dicha información es confirmado en el estudio Montero y Serra, debido a que refieren que pocos estudios han documentado los efectos de este tipo de ejercicio, aunado a ello, no hay ninguna prueba de la flexibilidad que se puede estimar en la mayoría de los ejercicios, así mismo es recomendable que se realice este tipo de ejercicio dos días por semana diez minutos por día con una intensidad moderada e incluyendo dicha actividad para el cuello, hombro, cadera, rodillas entre otros ${ }^{(12,38)}$.

En relación con lo anterior, se realizó una intervención basado en estos tipos de ejercicio a 128 mujeres mayores de 75 años en ensayo clínico de Japón, cuyo resultado indicó que la masa muscular y la extensión de rodilla no fue diferentemente significativo, pero sí mejora la velo- cidad y tiempo de marcha ${ }^{(24)}$. Otro estudio señaló que el trabajar durante 48 semanas con adultos mayores entrenamiento de extensores de rodilla ocasiona cambios en la fuerza y extensión de la rodilla, además refirieron hay que aumentar la dosis de manera semanal para alcanzar una hipertrofia miofibra de manera progresiva ${ }^{(40)}$.

\section{Ejercicios aeróbicos}

Un ejercicio aeróbico requiere del trabajo de varios grupos musculares para aumentar el número de capilares por fibra muscular, dichos cambios mejoran el fluido sanguíneo en los músculos ${ }^{(39)}$. La realización de actividades son un ejemplo el ciclismo, caminata, natación, subir escaleras, pedaleo de bicicleta estática, baile, gimnasia aeróbica y yoga, siendo ideal para los adultos mayores ${ }^{(28)}$.

Se recomienda una intensidad media, así como realizar precalentamiento de 10-15 minutos comenzando después con ejercicios de baja intensidad, se deben realizar entre 3 y 7 veces por semana con duración de 20-60 minutos y a un $40-60 \%$ de la frecuencia cardiaca máxima (8). Es oportuno mencionar que el ejercicio aeróbico no mejora el equilibrio, por lo tanto, es necesario recomendar a los AM con dificultad de movilidad que inicien por aumentar su fuerza y su equilibrio antes de emprender este tipo de ejercicio ${ }^{(9)}$.

Por otra parte, otro de los beneficios, es la mejora de la fuerza y flexibilidad de los extremidades inferiores, equilibrio, capacidad aeróbica y confianza para deambular ${ }^{(41)}$. Lo anterior se puede observar en un estudio realizado en Alemania a mujeres entre 65 y 80 años, intervinieron en este grupo mediante la danza aeróbica con una duración de 18 semanas. Los resultados indicaron que tuvo un efecto positivo de un programa de ejercicio polivalente en composición corporal, riesgo de caídas y factores de riesgo de enfermedad coronaria ${ }^{(42)}$.

\section{CONCLUSIÓN}

El ejercicio físico permite mejorar la salud del AM, además atenúa los cambios ocasionados por la sarcopenia, como la disminución de la masa muscular ${ }^{(39)}$. De acuerdo con la literatura las intervenciones de ejercicios en adultos mayores se observan cambios significativos en la fuerza muscular, mejoramiento de la función física, masa muscular, extensión de la rodilla, marcha, equilibrio, así como en la velocidad de marcha. Con respecto a los tipos de ejercicio el que ha reportado más beneficios fue el entrenamiento por fuerza, debido a que el aumento de masa muscular permite la disminución del riesgo. Así mismo, los ejercicios de vibración sirven para mejorar el equilibrio funcional y extensión de la rodilla.

En relación con los entrenamientos flexibilidad, equilibrio y aeróbicos ayudan al equilibrio funcional para la reducción del riesgo de caídas, velocidad de la marcha, así como factores de riesgo de enfermedad coronaria. Con 
base a lo anterior, es necesario realizar intervenciones integrales y multidisciplinarias en la atención de la sarcopenia, es por ello que el profesional de enfermería en la atención primaria debe de intervenir para la prevención de riesgo y mejorar la calidad de vida de los AM.

\section{REFERENCIAS BIBLIOGRÁFICAS}

1. Arias Proaño SC, Gutiérrez Verdugo DE. Relación entre la ingesta de proteína con la masa muscular en adultos mayores que asisten al programa 60 y piquito de San José en la Parroquia de conocoto comprendido desde enero hasta septiembre del 2015 [Tesis de licenciatura]. [Quito (ECU)]; Pontifica Universidad Católica de Ecuador; 2015. $89 \mathrm{p}$.

2. Cruz Jentoft AJ, Baeyens JP, Bauer JM, Boirie Y, Cederholm T, Landi F, et al. Sarcopenia: consenso europeo sobre su definición y diagnóstico Informe del Grupo europeo de trabajo sobre la sarcopenia en personas de edad avanzada. Age and egeing. 2010;39(4):412-23.

3. Cruz-Jentoft A, Stout J, Rueda R. La sarcopenia un problema frecuente y grave. Nutr Hosp. 2011;13-5.

4. Morley JE. Sarcopenia in the elderly. Fam Pract. 2012;29(Suppl 1):144-8.

5. Von Haehling S, Morley JE, Anker SD. An overview of sarcopenia: Facts and numbers on prevalence and clinical impact. J Cachexia Sarcopenia Muscle. 2010;1(2):129-33.

6. Shafiee G, Keshtkar A, Soltani A, Ahadi Z, Larijani B, Heshmat R. Prevalence of sarcopenia in the world: a systematic review and meta- analysis of general population studies. J Diabetes Metab Disord [Internet]. 2017 [Citado 26 Septiembre 2017];16:21. Disponible en: http://jdmdonline.biomedcentral.com/articles/10.1186/s40200017-0302-x.

7. Gómez-García S, Vega-Pérez ML, Tamez-Rodríguez AR, Guzmán-Pantoja JE. Fortalecimientodelaatención primaria del adulto mayor ante la transición demográfica en México. Atención Primaria [Internet]. 2013 [Citado 26 Septiembre 2017];45(5):231-2. Disponible en: http://ac.els-cdn. com/S0212656713000395/1-s2.0-S0212656713000395main.pdf?_tid $=144 \mathrm{f} 3 \mathrm{c} 6 \mathrm{e}-\mathrm{bccf}-11 \mathrm{e} 6-\mathrm{a} 1 \mathrm{~b} 2-00000 \mathrm{aab}-$ of27\&acdnat $=1481151006 \_1$ f8a601 cefa90c6b4d3d$844 \mathrm{bf} 3 \mathrm{cf} 47 \mathrm{ba}$

8. Del Rey Rozas T. Síndrome De Sarcopenia [Tesis de licenciatura]. [Santander (ESP)]; Universidad de Cantabria; 2014. $46 \mathrm{p}$.

9. Organización Mundial de la Salud. Informe mundial sobre el envejecimiento y la salud. Ginebra: OMS; c2015.
$282 \mathrm{p}$.

10. Sarabia-Cobo CM, Pérez-Rugosa V, Hermosilla-Grijalbo C, Núñez-García MJ, de Lorena Quintal P. Prevalencia de sarcopenia en mayores con demencia institucionalizados. Metas de Enfermería. 2015;18(6):17-21.

11. Plaza-Carmona M, Martínez-González L. Sarcopenia, envejecimiento y actividad física. Metas de Enfermería. 2015;18(2):69-73.

12. Ortiz-Diago PA, Morales-Mejía M. La importancia del ejercicio físico para el tratamiento de la sarcopenia [Tesis de licenciatura]. [Santiago de Cali (COL)]; Universidad del Valle; 2013. 86 p.

13. Padilla-Colón CJ, Sánchez-Collado P, Cuevas MJ. Beneficios del entrenamiento de fuerza para la prevención y tratamiento de la sarcopenia. Nutr Hosp [Internet]. 2017 [Citado 02 Octubre 2017]; 29(5):979-88. Disponible en: http://www.ncbi.nlm.nih.gov/pubmed/24951975

14. Casas-Herrero A, Izquierdo M. Ejercicio físico como intervención eficaz en el anciano frágil. An Sist Sanit Navar. 2012;35(1):69-85.

15. Masanés-Torán F, Navarro-López M, Sacanella-Meseguer E, López Soto A. ¿Qué es la sarcopenia? Semin Fund Esp Reumatol [Internet]. 2010 [Citado 04 Octubre 2017];11(1):14-23. Disponible en: http://linkinghub.elsevier.com/retrieve/pii/S1577356609000128

16. Paladines B, Quizhpi M, Villota P. Tratamiento integral de la sarcopenia senil. Rev la Fac Ciencias Químicas. 2016;(Ed. especial):41-8.

17. Molina JC. Sarcopenia en la pérdida funcional: rol del ejercicio. Rev Hops Clín Univ Chile [Internet]. 2008 [Citado 04 Octubre 2017];19(7):302-8. Disponible en: https:// www.redclinica.cl/Portals/0/Users/014/14/14/Publicaciones/Revista/sarcopenia.pdf

18. Palop Montoro MV, Párraga Montilla JA, Lozano Aguilera $\mathrm{E}$, Arteaga Checa M. Intervención en la sarcopenia con entrenamiento de resistencia progresiva y suplementos nutricionales proteicos. Nutr Hosp. 2015;31(4):1481-90.

19. Zuñiga R, Barrientos M. Conceptos básicos sobre obesidad sarcopénica en el adulto mayor. Rev Clínica Esc Med UCR-HSJD [Internet]. 2015 [Citado 07 Octuvre 2017];5(3):1-7. Disponible en: https://revistas.ucr.ac.cr/ index.php/clinica/article/view/19919

20. Richards EA. The Evolution of Physical Activity Promotion. Am J Nurs. 2015;115(8):50-4.

21. Ginarte Paúl M, Santiesteban Molina R. Importancia del ejercicio físico en la salud del adulto mayor. Rev Cuba Med Física y Rehabil. 2012;4(2):125-34. 
22. Heredia Elvar JR, Donate Fl, Mata F, García-Orea G, Segarra Nunez V, Moral S. Sarcopenia, Obesidad Sarcopénica y Papel del Ejercicio Físico. PublicE. 2013.

23. Hibbert JE, Jones TE, Domire ZJ. Change in Acute Response of Aged Women to Resistance Exercise Following a Long-Duration Stretching Intervention. The FASEB Journal [Internet]. 2017 [Citado 14 Octubre 2017];31(Suppl 1). Disponible en: http://www.fasebj.org/content/31/1_Supplement/839.25.abstract

24. Kim H, Suzuki T, Saito K, Yoshida H, Kojima N, Kim $M$, et al. Effects of exercise and tea catechins on muscle mass, strength and walking ability in community-dwelling elderly Japanese sarcopenic women: A randomized controlled trial. Geriatr Gerontol Int. 2013;13(2):458-65.

25. Bulechek G, Bulecher H, Dochterman J, Wagner C. Clasificación de intervenciones de enfermería (NIC). 6a ed. España: Elsevier; c2014. 664 p.

26. Ávila-Funes JA, García-Mayo EJ. Beneficios de la práctica del ejercicio en los ancianos. Gac Med Méx. 2004;140(4):431-6.

27. Mata-Ordoñez F, Chulvi-Medrano I, Heredia-Elvar JR, Moral-González S, Marcos-Becerro JF, Da Silva-GrigogoIleto ME. Entrenamiento de la fuerza y sarcopenia. Evidencias actuales. J Sport Health Res. 2013;5(1):7-24.

28. Paccini Lustosa L, Gomes Pereira DA, Netto Parentoni A, Correa Dias R, Domingues Dias JM, Máximo Pereira LS. Impact of Aerobic Training Associated with Muscle Strengthening in Elderly Individuals at Risk of Sarcopenia: A Clinical Trial. J Gerontol Geriatr Res. 2015;4(2):10-3.

29. Bellomo RG, lodice P, Maffulli N, Maghradze T, Coco $V$, Saggini R. Muscle strength and balance training in sarcopenic elderly: A pilot study with randomized controlled trial. Eur J Inflamm. 2013;11(1):193-201.

30. Kim H, Kim M, Kojima N, Fujino K, Hosoi E, Kobayashi $\mathrm{H}_{\text {, et }}$ al. Exercise and Nutritional Supplementation on Community-Dwelling Elderly Japanese Women With Sarcopenic Obesity: A Randomized Controlled Trial. J Am Med Dir Assoc. 2016;17(11):1011-9.

31. Palop Montoro MV, Párraga Montilla JA, Lozano Aguilera E, Arteaga Checa M. El entrenamiento vibratorio como intervención en la sarcopenia: Repercusiones en el sistema neuromuscular de los adultos mayores. Nutr Hosp. 2015;32(4):1454-61.

32. Santin-Medeiros F, Garatachea Vallejo N. Efectos musculoesqueléticos del entrenamiento con vibraciones en ancianos. Rev Esp Geriatr Gerontol. 2010;45(5):281-4.

33. Verschueren SM, Bogaerts A, Delecluse $C$, Claessens $A L$, Haentjens $P$, Vanderschueren $D$, et al. The effects of whole-body vibration training and vitamin $\mathrm{D}$ supplemen- tation on muscle strength, muscle mass, and bone density in institutionalized elderly women: A 6-month randomized, controlled trial. J Bone Miner Res. 2011;26(1):42-9.

34. Gómez-Cabello A, González-Agüero A, Ara I, Casajús JA, Vicente-Rodríguez G. Efectos de una intervención de vibración corporal total sobre la masa magra en personas ancianas. Nutr Hosp. 2013;28(4):1255-8.

35. Wei N, Pang MY, Ng SS, Ng GY. Optimal frequency/time combination of whole-body vibration training for improving muscle size and strength of people with age-related muscle loss (sarcopenia): A randomized controlled trial. Geriatr Gerontol Int. 2017;17(10):1412-20.

36. Hernández-Rodríguez J, Licea-Puig ME. Generalidades y tratamiento de la Sarcopenia. Revistas Médicas UIC. 2017;30(2):71-81.

37. Chodzko-Zajko WJ, Proctor DN, Fiatarone Singh MA, Minson CT, Nigg CR, Salem GJ, et al. Exercise and physical activity for older adults. Med Sci Sports Exerc. 2009;41(7):1510-30.

38. Montero- Fernández N, Serra-Rexach JA. Role of exercise on sarcopenia in the elderly. Eur J Phys Rehabil Med. 2013;49(1):131-43.

39. Realpe Villagomez LE. Tendencia actual del tratamiento de la sarcopenia en el adulto mayor [Trabajo de grado]. [Bogotá (COL)]; Pontifica Universidad Javeriana; 2014. $84 \mathrm{p}$.

40. Bickel CS, Cross JM, Bamman MM. Exercise dosing to retain resistance training adaptations in young and older adults. Med Sci Sports Exerc. 2011;43(7):1177-87.

41. Vidarte Claros JA, Quintero Cruz MV, Beltrán YH. Efectos del ejercicio físico en la condición física funcional y la estabilidad en adultos mayores. Hacia la Promoción la Salud. 2012;17(2):79-90.

42. Kemmler W, von Stengel S, Engelke K, Häberle L, Mayhew $\mathrm{JL}$, Kalender WA. Exercise, body composition, and functional ability. a randomized controlled trial. Am J Prev Med. 2010;38(3):279-87. 